Reprod. Nutr. Dévelop., 1982, 22 (1 A), 75-80.

\title{
Dérivation de la bile dans l'iléon terminal chez le porc : effet sur le niveau de sécrétion des acides biliaires et sur I'utilisation digestive du régime
}

\author{
Catherine JUSTE, T. CORRING \\ avec la collaboration technique de J. P. HAUTDUCOEUR, R. LEVREL et G. DUCHASTEL \\ Laboratoire de Physiologie de la Nutrition \\ I.N.R.A., 78350 Jouy-en-Josas, France.
}

Summary. Diversion of bile into the distal ileum in pig : effects on bile acid output and digestive utilization of the diet.

Bile salt output and digestive utilization of a high-lipid diet were studied in pig bile collected from the common bile duct and infused into the duodenum then into the distal ileum of the animal.

Five Large-White pigs of $50 \mathrm{~kg}$ body-weight were fitted with three permanent fistulae, one in the common bile duct for complete collection of bile secretion, and the other two in the duodenum and the ileum (at about $80 \mathrm{~cm}$ from the ileo-caecal valve) for returning the secretion to the animal.

The diversion of the bile into the distal ileum led to a significant 81 p. 100 decrease in daily bile acid output and a 36 p. 100 decrease in apparent digestibility of dietary energy when compared with values obtained when bile was infused into the duodenum. The latter decrease was mainly due to a marked diminution ( $-60.4 \mathrm{p} .100)$ of the digestive utilization of the dietary lipids.

The results show the role of the pig small intestine in maintaining an efficient enterohepatic circulation of bile acid as a result of optimal utilization of the dietary lipids.

\section{Introduction.}

Chez le porc (Corring et al., 1979), la dérivation totale de la sécrétion biliaire hors de la lumière intestinale entraîne une diminution de l'utilisation digestive des lipides totaux sans affecter la digestibilité apparente des glucides et de l'azote. Cette diminution, de l'ordre de 52 p. 100, est due à l'absence des lipides biliaires dans l'intestin puisque la réintroduction de la partie électrolytique de la bile ne rétablit pas l'utilisation digestive à des valeurs comparables à celles obtenues lorsque la sécrétion biliaire est restituée à l'animal au niveau duodénal.

Selon Morgan et Simmonds (1962) chez le rat, la présence des lipides biliaires dans la lumière intestinale ne serait pas indispensable à l'hydrolyse des triglycérides alimentaires et à l'absorption des produits libérés. Le facteur essentiel serait la 
présence au niveau sanguin et au niveau intracellulaire intestinal des acides biliaires, donc la nécessité de maintenir intact un recyclage entérohépatique.

On sait que les sels biliaires sont réabsorbés dans la partie distale de l'intestin grêle chez plusieurs espèces. Ainsi, chez le rat (Dietschy, Salomon et Siperstein, 1966), l'absorption est 4 fois plus intense sur le dernier dixième du grêle que sur le premier. Des valeurs voisines ont été rapportées chez le chien, et chez le lapin la capacité d'absorption du taurocholate est 57 fois plus importante dans l'iléon terminal que dans le duodéno-jéjunum (Glasser, Weiner et Lack, 1965). Enfin, chez I'homme (Heaton et al., 1968 ; Meihoff et Kern, 1968), le dernier quart de l'intestin grêle est principalement responsable de la réabsorption des acides biliaires. Aucune donnée comparable n'existe chez le porc.

Le travail rapporté a pour but l'étude de l'utilisation digestive d'un régime riche en lipides lorsque la bile n'irrigue que la partie distale de l'iléon afin de maintenir le recyclage entérohépatique des lipides biliaires, en supposant que la réabsorption des sels biliaires chez le porc se fait préférentiellement à ce niveau comme chez les autres espèces citées. Le niveau du recyclage entérohépatique a été estimé en mesurant la quantité d'acides biliaires sécrétés dans la bile recueillie et en la comparant à celle obtenue lorsque la bile est réintroduite dans le duodénum, chez le même animal.

\section{Matériel et méthodes.}

Animaux et régime. - Cinq porcs Large White, mâles castrés, de poids vif moyen $50 \mathrm{~kg}$ avant l'intervention chirurgicale, sont utilisés. Le régime expérimental, dont la composition est rapportée dans le tableau 1, contient 22,7 p. 100 de lipides en matière sèche. Les pourcentages de cellulose et de vermiculite (silicate)

TABLEAU 1

Composition du régime expérimental

\begin{tabular}{|c|c|c|c|c|}
\hline Composants & $\mathrm{g} / 100 \mathrm{~g}$ & & & \\
\hline Farine de poisson (1) & 23 & Matière sèche $93,8 \%$ & & \\
\hline Huile d'arachide . . . . . . & 21 & Protéines $(N \times 6,25))$ & & 15,8 \\
\hline Amidon de maïs $\ldots \ldots \ldots \ldots \ldots \ldots \ldots \ldots$ & 20 & Lipides totaux & en \% M.S. & 22,7 \\
\hline Cellulose $\ldots \ldots \ldots \ldots \ldots \ldots \ldots \ldots$ & 15 & Energie $(\mathrm{kcal} / \mathrm{kg})$ & $\int \infty$ & 4700 \\
\hline Vermiculite $\ldots \ldots \ldots \ldots \ldots \ldots \ldots \ldots$ & 17,5 & & & \\
\hline Mélange minéral $(2) \ldots \ldots \ldots \ldots \ldots \ldots$ & 2,5 & & & \\
\hline Mélange vitaminique ${ }^{(3)} \ldots \ldots \ldots \ldots \ldots$ & 1 & & & \\
\hline
\end{tabular}

(1) 68,6 p. 100 de protéines. (2) En p. 100 du régime : phosphate dicalcique : 1,25; craie broyée : 0,19 ; chlorure de potassium : 0,50 ; carbonate de magnésie : 0,06 ; oligo-éléments en $\mathrm{mg} / \mathrm{kg}$ aliment : $\mathrm{FeSO}_{4}, 7 \mathrm{H}_{2} \mathrm{O}: 480 ; \mathrm{MnSO}_{4}, \mathrm{H}_{2} \mathrm{O}: 144 ; \mathrm{CuSO}_{4}, 5 \mathrm{H}_{2} \mathrm{O}: 120 ; \mathrm{ZnSO}_{4}, \mathrm{H}_{2} \mathrm{O}: 270 ; \mathrm{KI}: 0,06$. (3) En $\mathrm{mg} / \mathrm{kg}$ aliment : acétate de rétinol (50 000 UI) : 100 ; cholécalciférol (100 000 UI) : $10 ; \alpha$-tocophérol (250 UI) : 160 ; vitamine $K: 4,4$; vitamine $B_{1}: 2,2$; vitamine $B_{2}: 2,6$; riboflavine :6,2; acide nicotinique $: 22$; acide folique : 1,6 ; vitamine $B_{12}(500 \mathrm{mg} / \mathrm{kg}): 66$; inositol : 400 ; biotine : 12 ; pantothénate de calcium : 20 ; choline $(50$ p. 100) : 2600 ; acide paraminobenzoïque : 44 . 
sont élevés afin de diluer l'apport énergétique dû à la forte teneur du régime en huile d'arachide. Les porcs reçoivent la même quantité d'aliment (1 $400 \mathrm{~g}$ matière sèche/jour) (méthode du paired-feeding de Mitchell, 1930), et les refus sont pesés après chacun des 3 repas journaliers, distribués respectivement à $7 \mathrm{~h}, 11 \mathrm{~h}$ et $15 \mathrm{~h}$.

Technique chirurgicale. - Après adaptation à la cage à métabolisme et au régime expérimental pendant 8 jours, les animaux sont équipés de 3 canules permanentes en silastic $\left({ }^{1}\right)$ médical. Deux d'entre elles sont placées respectivement dans le canal cholédoque pour collecter la bile et dans le duodénum pour restituer la sécrétion à l'animal selon la technique décrite antérieurement (Juste, Corring et Breant, 1979). La troisième servant également à la restitution de la bile ( $\varnothing$ extérieur : $3,18 \mathrm{~mm}$; $\varnothing$ intérieur : $1,57 \mathrm{~mm}$ ) est introduite dans l'iléon en direction aborale et à $100 \mathrm{~cm}$ environ de la valvule iléo-cæacale.

Schéma expérimental. - Après l'intervention chirurgicale, les animaux sont soumis à une période de récupération post-opératoire de 10 jours. La bile cholédocienne (collectée par la canule placée dans le canal cholédoque) est intégralement retournée dans le duodénum pendant cette période, chez tous les animaux, au moyen d'une pompe péristaltique dont le débit est réglé en fonction des heures de distribution des repas (Juste, Corring et Breant, 1979).

Les animaux sont ensuite soumis à 2 périodes consécutives de 8 jours chacune :

1) Période témoin : la bile recueillie est réintroduite dans le duodénum.

2) Période expérimentale : la bile recueillie est réintroduite dans l'iléon.

Les digestibilités apparentes (matière organique, énergie totale, azote et lipides totaux), les rétentions (azote et énergie) ainsi que la quantité des acides biliaires sécrétés sont mesurées au cours des 5 derniers jours de chacune des 2 périodes. Les méthodes utilisées pour ces différentes mesures ont été décrites précédemment (Corring et al., 1979 ; Juste, Corring et Breant, 1979).

Enfin, la distance séparant la canule iléale de la valvule iléo-cæcale est mesurée sur tous les animaux après abattage.

\section{Résultats.}

Les résultats rapportés ont été calculés à partir des données obtenues sur les 5 animaux opérés et observés sur un total de 50 journées expérimentales, dont 25 en restitution biliaire duodénale et 25 en restitution iléale. La canule iléale était implantée à $81 \pm 3 \mathrm{~cm}$ de la valvule iléo-cæcale.

1. Effet d'une dérivation biliaire dans l'iléon terminal sur la quantité d'acides biliaires sécrétés dans la bile cholédocienne. - La dérivation de la bile dans l'iléon terminal conduit à une réduction très importante de la quantité de sels biliaires

(1) Dow Corning Corporation medical Products, Midland, Michigan, 48640 USA. 
sécrétés par jour : $-81,5$ p. $100(P<0,001$; fig. 1), par rapport au niveau quotidien observé pendant la période témoin, en conditions de restitution biliaire duodénale.

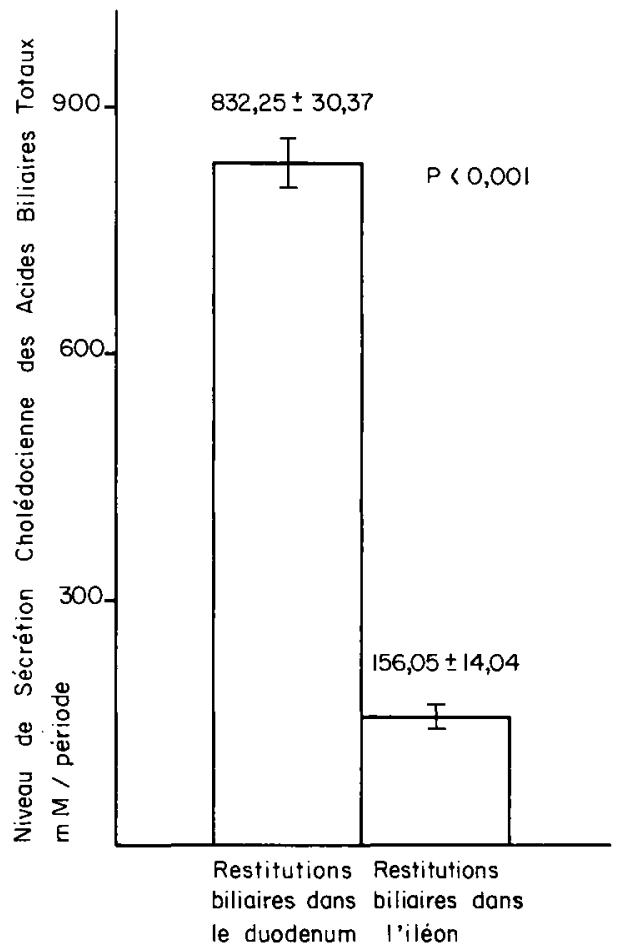

FIG. 1. - Effet d'une dérivation biliaire dans f'iléon terminal sur le niveau de sécrétion cholédocienne des acides biliaires chez le porc.

TABLEAU 2

Effet d'une dérivation biliaire dans l'iléon terminal sur la digestibilité et la rétention du régime chez le porc

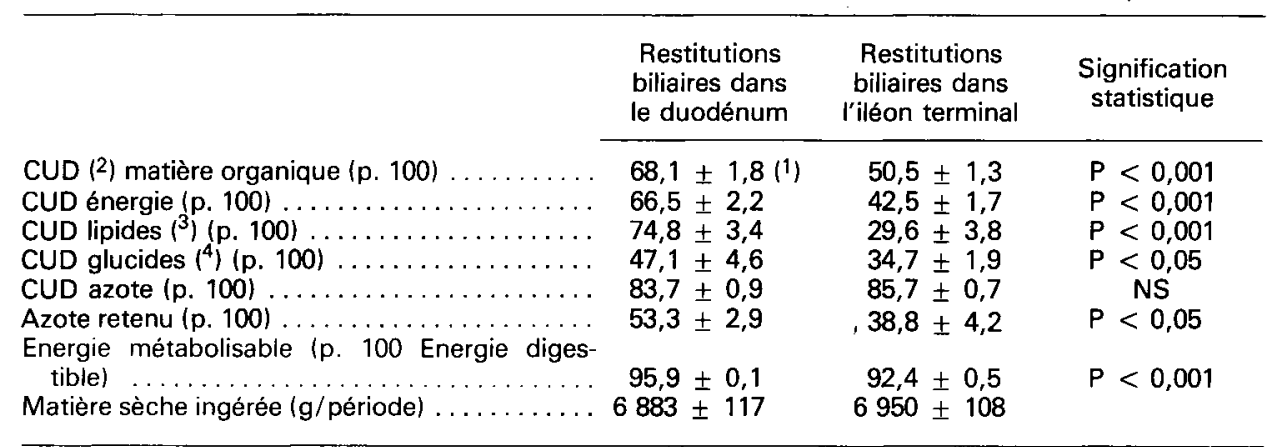

( $\left.{ }^{1}\right)$ Moyenne \pm SEM. $\left({ }^{2}\right)$ Coefficient d'utilisation digestive. $\left({ }^{3}\right)$ Lipides totaux. $\left({ }^{4}\right)$ Energie provenant des glucides.

Analyse statistique : analyse de variance et test de Student. 
2. Effet d'une dérivation biliaire dans l'iléon terminal sur l'utilisation digestive du régime et la rétention azotée. - Pour chacun des critères étudiés, 2 moyennes sont calculées, l'une relative à la période témoin (restitution biliaire dans le duodénum) l'autre relative à la période expérimentale (restitution biliaire iléale). Les résultats ainsi que leur signification statistique sont rapportés dans le tableau 2.

En se référant aux valeurs déterminées au cours de la période témoin, on constate que la dérivation de la bile dans l'iléon terminal entraîne une diminution hautement significative $(P<0,001)$ des digestibilités apparentes de la matière organique $(-25,84$ p. 100), de l'énergie totale $(-36,09$ p. 100), des lipides totaux (-60,43 p. 100). La digestibilité apparente de l'énergie provenant des glucides et la rétention azotée sont également affectées par la dérivation de bile dans l'iléon (respectivement : $-26,33$ p. 100 et $-27,20$ p. 100) mais le seuil de signification statistique est cependant réduit $(P<0,05)$. Enfin, la digestibilité apparente de l'azote n'est pas significativement affectée par l'absence de bile dans la lumière de la partie proximale de l'intestin grêle.

\section{Discussion.}

Le but du travail rapporté ici était l'étude de l'utilisation digestive d'un aliment riche en lipides chez le porc privé de sa sécrétion biliaire dans la partie proximale de l'intestin grêle. Afin de réaliser un modèle expérimental adéquat et de maintenir le recyclage entérohépatique des sels biliaires jugé essentiel dans la digestion et l'absorption des lipides chez le rat (Morgan et Simmonds, 1962), la bile a été collectée avant son entrée dans le duodénum et réintroduite à moins d'un mètre de la valvule iléo-cæcale. Les résultats obtenus montrent que dans ces conditions expérimentales, la quantité de sels biliaires sécrétés dans la bile cholédocienne est fortement diminuée ( -81 p. 100), par rapport au niveau observé chez le même animal au cours d'une période témoin (réintroduction de la bile dans le duodénum). Au cours d'une expérience précédente (Juste et Corring, 1979) il a été montré qu'une interruption totale du recyclage entérohépatique réduit de 80 p. 100 la quantité d'acides biliaires sécrétés dans la bile cholédocienne, chez le porc. Cependant, la teneur en lipides du régime utilisé était environ 10 fois plus faible que celle de l'aliment distribué dans l'expérience présente. De ce fait, on ne peut affirmer que la dérivation de la bile dans la partie terminale de l'iléon conduit à l'absence totale de recyclage entérohépatique. Toutefois, les résultats suggèrent une malabsorption des sels biliaires sinon totale, du moins très importante lorsque la bile n'irrigue que les 80 derniers centimètres du grêle et le gros intestin chez le porc. On peut souligner à ce stade que la partie terminale de l'iléon ne paraît pas être le site d'absorption préférentiel des sels biliaires chez le porc comme elle l'est chez d'autres espèces telles que le rat (Dietschy, Salomon et Siperstein, 1966), le chien et le lapin (Glasser, Weiner et Lack, 1965).

Les résultats obtenus dans l'étude rapportée montrent également une diminution sévère de la digestibilité apparente de l'énergie totale qui peut s'expliquer pour 85 p. 100 par la diminution de la digestibilité apparente des lipides totaux. En effet, la digestibilité apparente des glucides est relativement peu affectée et celle 
de l'azote non modifiée. Les résultats obtenus par Morgan et Simmonds (1962) chez le rat sont fondamentalement différents. Ces auteurs ont montré que l'absorption lipidique est diminuée de 59 p. 100 lorsque la bile est exclue du tractus digestif et dérivée dans la voie urinaire, et ce par rapport aux valeurs obtenues quand la bile est réintroduite dans le duodénum. Par contre, l'absorption des lipides n'est réduite que de 24 p. 100 lorsque la sécrétion biliaire est infusée dans les 2 derniers centimètres de l'iléon. II est cependant regrettable que le niveau de sécrétion des acides biliaires dans la bile cholédocienne n'ait pas été mesurée par les auteurs dans ce cas de restitutions biliaires.

Si la digestibilité apparente de l'azote n'est pas significativement affectée par la dérivation de la bile dans l'iléon terminal, on constate en revanche que la rétention azotée est diminuée de $22 \mathrm{p} .100$. Une chute de rétention azotée avait déjà été observée lors de l'interruption totale du recyclage entérohépatique (Corring et al., 1979). Cela peut s'expliquer en premier lieu par la diminution de l'apport d'énergie métabolisable (- 38 p. 100), qui devient alors un facteur limitant pour la rétention azotée. En second lieu, il est vraisemblable qu'une perte non négligeable d'électrolytes, consécutive à la dérivation biliaire dans l'iléon terminal, contribue à la diminution de la rétention azotée (Terroine et Reichert, 1929 ; Corring et al., 1979).

Compte tenu du but initial de la présente étude, nous sommes amenés à conclure que le modèle expérimental proposé n'est pas adéquat. II ne permet pas le maintien du recyclage entérohépatique des sels biliaires, vraisemblablement par défaut de leur réabsorption dans la partie terminale de l'iléon et le gros intestin.

Reçu en avril 1981.

Accepté en août 1981.

\section{Références}

CORRING T., JUSTE C., SIMOES-NUNES C., BOURDON D., 1979. Effet de la sécrétion biliaire sur la digestion chez le porc. Ann. Biol. anim. Bioch. Biophys., 19, 1123-1130.

DIETSCHY J. M., SALOMON H. S., SIPERSTEIN M. D., 1966. Bile acid metabolism. I. Studies on the mechanisms of intestinal transport. J. clin. Invest., 45, 832-846.

GLASSER J. E., WEINER I. M., LACK L., 1965. Comparative physiology of intestinal taurocholate transport. Am. J. Physiol., 208, 359-362.

HEATON K. W., AUSTAD W. I., LACK L., TYOR M. P., 1968. Enterohepatic circulation of $\mathrm{C}^{14}$-labeled bile salts in disorders of the distal small bowel. Gastroenterology, 55, 5-16.

JUSTE C., CORRING T., 1979. Effet d'une interruption et de restitutions partielles du recyclage entérohépatique sur le niveau de l'excrétion biliaire chez le porc. Ann. biol. anim. Bioch. Biophys., 19, 405-412.

JUSTE C., CORRING T., BREANT Ph., 1979. Excrétion biliaire chez le porc : niveau et réponse au repas. Ann. Biol. anim. Bioch. Biophys., 19; 79-90.

MEIHOFF W. E., KERN F., 1968. Bile salt malabsorption in regional ileitis, ileal resection, and mannitol-induced diarrhea. J. clin. Invest., 47, 261-267.

MITCHELL H. H., 1930. The paired feeding method: its value and limitations in live-stock experimentation. Proc. am. Soc. anim. Prod., 63-73.

MORGAN R. G. H., SIMMONDS W. J., 1962. The relative effects of diversion of bile to the ileum or to the urinary bladder on fat absorption and gastrointestinal motility in the rat. $Q$. J. exp. Physiol., 47, 352-359.

TERROINE E. F., REICHERT T., 1929. Influence de la ration saline sur la grandeur de la rétention azotée au cours de la croissance. C. R. Acad. Sci. Paris, 188, 1268-1271. 